\title{
Applications of Nonstandard Finite Difference Schemes
}


This page is intentionally left blank 


\section{Applications of Nonstandard Finite Difference Schemes}

Editor

Ronald E. Mickens

Callaway Professor of Physics

Clark Atlanta University, Atlanta, Georgia 


\section{Published by}

World Scientific Publishing Co. Pte. Ltd.

P O Box 128, Farrer Road, Singapore 912805

USA office: Suite 1B, 1060 Main Street, River Edge, NJ 07661

UK office: 57 Shelton Street, Covent Garden, London WC2H 9HE

\section{British Library Cataloguing-in-Publication Data}

A catalogue record for this book is available from the British Library.

\section{APPLICATIONS OF NONSTANDARD FINITE DIFFERENCE SCHEMES}

Copyright $\odot 2000$ by World Scientific Publishing Co. Pte. Ltd.

All rights reserved. This book, or parts thereof, may not be reproduced in any form or by any means, electronic or mechanical, including photocopying, recording or any information storage and retrieval system now known or to be invented, without written permission from the Publisher.

For photocopying of material in this volume, please pay a copying fee through the Copyright Clearance Center, Inc., 222 Rosewood Drive, Danvers, MA 01923, USA. In this case permission to photocopy is not required from the publisher.

ISBN 981-02-4133-X

Printed in Singapore by Uto-Print 


\section{Preface}

Nearly two decades ago, I initiated a research program to understand the types and properties of numerical instabilities that exist in the construction of finite difference schemes for differential equations. The resulting procedures are now labeled nonstandard finite difference methods. Currently, other researchers are applying these techniques to obtain numerical solutions to a variety of differential equations that appear in many problems of interest to the natural and engineering sciences. An important feature of nonstandard schemes is that they often can provide numerical integration techniques for which the elementary numerical instabilities do not occur. While much theoretical work needs to be done on these methods to fully understand why they work, when they do work, etc., practical applications do exist in which the power of nonstandard schemes is clearly illustrated.

This edited volume gives expanded versions of invited talks that were presented in a minisymposium at the 1999 SIAM Annual Meeting held in Atlanta, Georgia. The minisymposium, "Nonstandard Finite Difference Schemes: Theory and Applications," consisted of four lectures by individuals actively working in this area. The main purpose of the minisymposium was to introduce the concept of nonstandard finite difference schemes and demonstrate their power and usefulness by applying these methods to several problems involving nonlinear convection-diffusion-reactions, electromagnetic scattering, and interacting population models of the LotkaVolterra type.

The book is organized into five chapters. The first gives a general review of nonstandard finite difference methods, certain rules for their construction, and the broad outline of how these rules can be applied to partic- 
ular ordinary and partial differential equations. In the second chapter, a new class of Eulerian-Lagrangian methods is developed that efficiently handle the numerically difficult advection-dominated transport problems with nonlinear reactions. The nonstandard techniques are based on a nonlocal numerical treatment of nonlinear reaction terms and more sophisticated discretizations of time derivatives. The numerical solution of the advection-reaction part of the problem is defined using an "exact" timestepping scheme. Standard finite differences are then used for solving the remaining diffusion part. The nonstandard numerical methods are relatively easy to implement and have much greater computational efficiency as compared to standard numerical methods. The relative importance of advection, and biological and chemical reactions are directly incorporated into the corresponding numerical scheme. Large time steps can be taken without affecting the accuracy of the numerical solutions. The nonstandard schemes are reliable and propagate sharp fronts accurately, even when the convection and reaction processes are highly dominant and the initial data are not small.

The third chapter illustrates the particularly successful application of nonstandard finite difference methods to wave propagation and scattering problems. These methods can be used to construct high accuracy algorithms to solve the wave equation and Maxwell's equations in two and three dimensions. For second order finite differences, the leading error term can be reduced from $O\left(h^{4}\right)$ to $O\left(h^{8}\right)$ simply by replacing the standard finite differences with nonstandard schemes. In two and three dimensions, the basic nonstandard concepts need to be generalized by modifying the standard finite differences' computational molecules. A discussion is given of the physical significance of this change and why it improves numerical stability.

In the fourth chapter, nonstandard discretization methods are used to develop a variety of discrete models for Lotka-Volterra type systems of ordinary differential equations. The main concern of the chapter is to study those numerical schemes that produce difference equations whose dynamics resemble that of their continuous counterparts. The resulting difference equations are said to be "dynamically consistent" with the corresponding differential equations if they both exhibit the same qualitative behavior such as stability, bifurcation, and (possible) chaotic properties. A very useful discussion is given on the distinction in procedures needed for asymptotically stable systems versus periodic systems. 
The last chapter is by Rita Meyer-Spasche. While she was not a participant in the SIAM minisymposium, her work on finite difference schemes is of such importance that I invited her to write a chapter for this volume. This chapter is concerned with the search for difference schemes which preserve, at moderate size of the time step, all important properties of a given differential equation. Exactness, stability properties, and symplecticity of various schemes are examined. In particular, a discussion is given on the conditions under which Runge-Kutta schemes are exact. This analysis provides new insights into the nature of these schemes.

As a contributor to and editor of this volume, I look forward to both personally extending the current knowledge of nonstandard schemes and for advances that will come from the efforts of others. While these schemes may not presently resolve all of the difficulties involved with finite difference models of differential equations, their use clearly gives in many cases much better discrete models than ones obtained using standard methods. My general view is that nonstandard schemes have an exciting future and will provide exciting opportunities for new results in pure mathematics and improved numerical solutions of differential equations.

Finally, I wish to thank my many colleagues for their interest in my work and for the various collaborations which have arisen. The following persons have been particularly helpful in these endeavors during the past several years: Matthias Ehrhardt, Universitöt des Saarlandes, GFR; Alicia Serfaty de Markus, Universidad de los Andes, Venezuela; Jesús VigoAguiar, Universidad de Salamanca, Spain. I am particularly grateful to Annette Rohrs for her editorial work involving the smooth integration of the various manuscripts into a document that led to this book. Without her efforts, this publishing project could not have been completed. Finally, I thank both DOE and the MBRS-SCORE Program at Clark Atlanta University for providing funds that allowed me to do research on nonstandard finite difference schemes.

Ronald E. Mickens

Atlanta, Georgia

December 1999 
This page is intentionally left blank 


\section{Contents}

Preface $\quad v$

Chapter 1 Nonstandard Finite Difference Schemes 1 Ronald E. Mickens

1.1 Introduction . . . . . . . . . . . . . . . 2

1.2 Exact Schemes . . . . . . . . . . . . . . . 5

1.3 Nonstandard Schemes . . . . . . . . . . . . . 19

1.4 Applications ....................... 23

1.4.1 First-Order Scalar ODE's . . . . . . . . . . . 23

1.4 .2 A Photoconduction Model . . . . . . . . . . . 26

1.4.3 The Duffing Oscillator . . . . . . . . . . . . . 29

1.4.4 Mixed Parity Oscillator . . . . . . . . . . . . . 31

1.4.5 A Cubic Reaction Problem in Neurophysiology . . . . . 34

1.4.6 Time-Independent Schrödinger Equations . . . . . . 35

1.4.7 Traveling Wave Solutions . . . . . . . . . . . 37

1.4.8 Linear Advection-Diffusion Equation . . . . . . . . . 41

1.4.9 A Combustion Model . . . . . . . . . . . . . . 42

1.4.10 Influence of Spatial Discretizations for PDE's . . . . . 44

1.5 Future Directions . . . . . . . . . . . . . . . 49

Bibliography $\quad 51$

Chapter 2 Nonstandard Methods for Advection-DiffusionReaction Equations

Hristo V. Kojouharov and Benito M. Chen

2.1 Introduction . . . . . . . . . . . . . 
2.2 Non-Standard Methods in One Dimension . . . . . . . . . . 58

2.2.1 Advection-Reaction Equations . . . . . . . . . 59

2.2.1.1 Logistic Growth Reaction Terms . . . . . . 59

2.2.1.2 Linear Reaction Terms . . . . . . . . . . 62

2.2.1.3 Nonlinear Reaction Terms . . . . . . . . . 64

2.2.2 Advection-Diffusion-Reaction Equations . . . . . . . 67

2.3 Error Analysis of the Non-Standard Method . . . . . . . . . 69

2.3.1 Advection-Reaction Equations . . . . . . . . . . 69

2.3.1.1 Zero Local Time-Truncation Error . . . . . 70

2.3.1.2 Zero Local Space-Truncation Error . . . . . . 71

2.3.1.3 Interpolation Errors . . . . . . . . . . . . 72

2.3.2 Advection-Diffusion-Reaction Equations . . . . . . 77

2.4 One-Dimensional Numerical Results . . . . . . . . . . . 84

2.4.1 Advection-Reaction Equations . . . . . . . . 85

2.4.2 Advection-Diffusion-Reaction Equations . . . . . . . 90

2.5 Non-Standard Methods in Multiple Dimensions . . . . . . . . 94

2.5.1 Development of the Non-Standard Method . . . . . . 94

2.5.2 Error Estimates . . . . . . . . . . . . . . 98

2.6 Summary . . . . . . . . . . . . . . . . . . 104

$\begin{array}{ll}\text { Bibliography } & 106\end{array}$

Chapter 3 Application of Nonstandard Finite Differences to Solve the Wave Equation and Maxwell's Equations 109 James B. Cole

3.1 Introduction . . . . . . . . . . . . . . . . . . 109

3.2 The One-Dimensional Wave Equation . . . . . . . . . . 111

3.2.1 Finite-Difference Time-Domain Algorithm . . . . . . . 111

3.2 .2 Algorithmic Error . . . . . . . . . . . . . . 115

3.2.3 Nonstandard Finite Differences . . . . . . . . . . . . 117

3.2.4 One-Dimensional Scattering . . . . . . . . . . . . 120

3.3 The Two and Three-Dimensional Wave Equation . . . . . 124

3.3.1 Standard FDTD Algorithm . . . . . . . . . . . . . 124

3.3.2 Generalized Nonstandard Finite Differences - Two Dimensions . . . . . . . . . . . . . . . . 125

3.2.3 Generalized Nonstandard Finite Differences in Three Dimensions . . . . . . . . . . . . . 130

3.4 Discretization and Stability $\ldots \ldots \ldots \ldots \ldots \ldots$ 
3.4.1 Discretization . . . . . . . . . . . . . 133

3.4 .2 Stability . . . . . . . . . . . . . . 133

3.5 NSFD Solution of Maxwell's Equations . . . . . . . . . . 139

3.5.1 The Standard Yee Algorithm . . . . . . . . . . . 139

3.5.2 Nonstandard Yee Algorithm . . . . . . . . . . . . 142

3.5.3 Maxwell's Equations in a Conducting Medium . . . . 146

3.5.4 Electromagnetic Simulations . . . . . . . . . . . 149

3.6 Summary . . . . . . . . . . . . . . . . 150

3.7 Conclusion $\ldots \ldots \ldots \ldots \ldots \ldots \ldots \ldots \ldots$

$\begin{array}{ll}\text { Bibliography } & 152\end{array}$

Chapter 4 Non-standard Discretization Methods for Some Biological Models

H. Al-Kahby, F. Dannan, and S. Elaydi

4.1 Introduction . . . . . . . . . . . . . . . . 155

4.2 Stability of Lotka-Volterra Differential Equations . . . . . . . 157

4.3 Classical Discretization . . . . . . . . . . . . . . 158

4.4 Nonstandard Discretization Schemes . . . . . . . . . . 160

4.5 Competitive and Cooperative Discrete Models . . . . . . . . . 161

4.6 Permanence of Discrete Competitive and

Cooperative Systems . . . . . . . . . . . . . . . . 164

4.7 Predator-prey Discrete Models . . . . . . . . . . . . 165

4.8 Global Stability of Competitive and

Cooperative Systems . . . . . . . . . . . . . 170

4.9 Leslie Predator-prey Model . . . . . . . . . . . . . . . 172

4.10 Other Nonstandard Numerical Schemes . . . . . . . . . . . 174

4.11 A Kolmogorov Model of Cooperative Systems . . . . . . . . 177

4.12 Open Problems . . . . . . . . . . . . . . . 178

$\begin{array}{ll}\text { Bibliography } & 179\end{array}$

Chapter 5 An Introduction to Numerical Integrators Preserving Physical Properties

Martin J. Gander and Rita Meyer-Spasche

5.1 Introduction . . . . . . . . . . . . . . . . 182

5.2 Exact Difference Schemes . . . . . . . . . . . . . . . . 184

5.2.1 Standard Numerical Schemes as Exact Schemes . . . . . 184 
5.2.1.1 First Order Schemes . . . . . . . . . 185

5.2.1.2 Second Order Schemes . . . . . . . . . 187

5.2.1.3 Higher Order Schemes . . . . . . . . . . 192

5.2.1.4 Runge-Kutta Schemes . . . . . . . . . 195

5.2.2 Functional Fitting RK-Methods . . . . . . . . . . . 199

5.2.3 Schemes for Given Differential Equations . . . . . . . . 203

5.2.3.1 Exact Schemes for Given Differential Equations 203

5.2.3.2 Nonstandard Schemes for Parabolic Equations with Blow-Up Solutions: Le-Roux Schemes . . 205

5.3 Dynamics of Difference Schemes . . . . . . . . . . . . . 208

5.3.1 Continuous Dynamical Systems . . . . . . . . . . . 208

5.3.2 Discrete Dynamical Systems _. . . . . . . . . . 210

5.3.3 Forward Euler Scheme . . . . . . . . . . . . . . . 212

5.3.4 Midpoint Euler Scheme . . . . . . . . . . . . . . 215

5.3.5 Linearly Implicit Euler Schemes _. . . . . . . . . . 219

5.3.5.1 Details of the Dynamics . . . . . . . . 221

5.3.5.2 Superstability . . . . . . . . . . . 223

5.3.6 The Linearly Implicit Lintrap Scheme . . . . . . . . . 224

5.3.6.1 Existence Intervals . . . . . . . . . . . 226

5.3.6.2 Adjoint and Self-Adjoint Schemes . . . . . . 227

5.3.6.3 Convergence of the Scheme . . . . . . . . 228

5.3.6.4 Stability . . . . . . . . . . . . 228

5.4 Symplectic and Energy-Conserving Schemes . . . . . . . . . 229

5.4.1 Canonical Hamiltonian Systems . . . . . . . . . 230

5.4.1.1 Symplectic Euler . . . . . . . . . . . 232

5.4.1.2 The Lintrap Scheme . . . . . . . . . . 234

5.4.2 Non-Canonical Hamiltonian Systems . . . . . . . . 236

5.4.2.1 Lintrap for Lotka-Volterra . . . . . . . . . . 238

5.4.2.2 Symplectic Euler for Lotka-Volterra . . . . . . 242

5.5 Acknowledgement . . . . . . . . . . . . 243

Bibliography 244

$\begin{array}{lll}\text { Appendix A } & \text { Other Relevant References } & 247\end{array}$

List of Contributors $\quad 249$ 